\title{
EDGE WAVES WITH LONGSHORE CURRENTS
}

\author{
BY \\ HUNG-CHU HSU \\ Tainan Hydraulics Laboratory, National Cheng Kung University, Tainan 701, Taiwan
}

\begin{abstract}
We present an exact solution to the nonlinear governing equations for nonlinear edge waves with an underlying longshore current, propagating over a planesloping beach. By performing an analysis in Lagrangian variables we describe the flow characteristics in great detail.
\end{abstract}

1. Introduction. Edge waves are trapped waves near the shoreline on a sloping beach since their amplitude is maximal at the shoreline and decays rapidly offshore. These three-dimensional waves propagate along the straight shoreline, and the waveform is sinusoidal in the longshore. The edge waves play an important role in the dynamics of coastal zone and beach erosion processes (Leblond and Mysak [1]).

The edge wave phenomenon has been extensively studied and discussed in the mathematical literature within the framework of linear theory. Due to the small displacements associated with these waves, the governing equations for water waves or the shallow-water equations are linearized, and this simplification permits a thorough analysis (Stokes [2], Lamb [3], Ursell [4], Ehrenmark [5]). Nonlinear corrections to the linear theory have been given by Whitham [6]. He showed the existence of irrotational weakly nonlinear edge waves that propagate parallel to the shore using a formal Fourier series expansion for the full water-wave theory. A study of properties of nonlinear progressive edge waves based on the fact that the evolution is described by the nonlinear Schrödinger equation was performed by Yeh [7]. Mok and Yeh [8] studied the mass transport of nonlinear progressive edge waves.

Some exact solutions describing nonlinear edge waves in the Lagrangian framework were also obtained. Yih [9] and Mollo-Christensen [10] found that the deep water-wave solution discovered by Gerstner [11] can be adapted to construct edge waves propagating along a plane-sloping beach. However, this treatment of the edge wave problem provides only an implicit form for the free water surface. Constantin [12 presented an explicit edge wave solution. This flow has been a starting point for a number of interesting studies within the class of gravity or geophysical edge waves (cf. Ehrnstrom et al. [13, Henry

Received December 21, 2013 and, in revised form, January 19, 2014.

2010 Mathematics Subject Classification. Primary 76B15, 35B36, 74 G05.

The author is grateful to the referee for several useful suggestions.

E-mail address: hchsu@thl.ncku.edu.tw 
and Mustafa [14, Johnson [15], Stuhlmeier [16, Matioc [17]). For a modern exposition of Gerstner's wave we refer to the discussion in Constantin [18] and Henry [19] for extensions to equatorial surface geophysical flows see Constantin [20, Constantin and Germain 21] and Hsu 22], while extensions relevant for geophysical internal waves are described in the recent papers (Constantin [23, 24]) and (Hsu [25, 26]). We would only like to emphasize the special nature of Gerstner's flow by pointing out that all particles perform a circular motion. This is in stark contrast to the situation encountered beneath Stokes waves (periodic irrotational travelling waves); cf. Constantin [27, Henry 28, Constantin and Strauss [29].

The inclusion of longshore currents for the linear edge wave is described by Howd et al. [30. The aim of the present paper is to extend the explicit exact solution in Constantin 12 to include an underlying uniform current. The solution is presented in Lagrangian coordinates by describing the circular path of each particle.

2. Governing equations. We take a plane beach and adopt a coordinate system as shown below, which shoreline being the $x$-axis and still sea in the region

$$
R=\left\{(x, y, z): x \in \mathbb{R}, y \leq b_{0}, 0 \leq z \leq\left(b_{0}-y\right) \tan \alpha\right\}
$$

for some $b_{0} \leq 0$; here $\alpha \in(0, \pi / 2)$ defines the uniform slope. Let $(u, v, w)$ be the velocity field, and the governing equations for the propagation of gravity waves are given by

$$
\left\{\begin{array}{l}
u_{t}+u u_{x}+v u_{y}+w u_{z}=-\frac{1}{\rho} P_{x}, \\
v_{t}+u v_{x}+v v_{y}+w v_{z}=-\frac{1}{\rho} P_{y}-g \sin \alpha, \\
w_{t}+u w_{x}+v w_{y}+w w_{z}=-\frac{1}{\rho} P_{z}-g \cos \alpha,
\end{array}\right.
$$

coupled with the equation of mass conservation

$$
\rho_{t}+u \rho_{x}+v \rho_{y}+w \rho_{z}=0
$$

and with the incompressibility constraint

$$
u_{x}+v_{y}+w_{z}=0 .
$$

Here $t$ stands for time, $g=9.81 \mathrm{~m} \mathrm{~s}^{-2}$ is the (constant) gravitational acceleration, and $\rho$ is the water's density, while $P$ is the pressure. The boundary conditions which select the water-wave problem from all possible solutions of the equations (2.1) and (2.3) are (Constantin [18, Johnson [31]): (i) The dynamic boundary condition $P=P_{0}$ at the free surface, where $P_{0}$ is the constant atmosphere pressure, decouples the motion of the air from that of the water. (ii) The kinematic boundary condition at the free surface expresses the fact that the same particles always form the free water surface. (iii) The bottom is assumed to be impermeable and the normal velocity component at the sloping bed to be zero. The general description of the propagation of a water wave is encompassed by the equations (2.1) and (2.3) and the three boundary conditions (i)-(iii), a distinctive feature being that the free surface is not known and must be determined as a part of the solution. 




FIG. 1. Cross section of the still sea.

3. An exact solution in the Lagrangian description. In this section, we will present the exact solution of the edge wave problem. This solution represents edge waves travelling parallel to the shoreline which has a constant speed of propagation $c_{0}$. Throughout this paper, the Lagrangian positions $(x, y, z)$ of the fluid particles are given as functions of labeling variables $(a, b, c)$ and time $t$ by

$$
\left\{\begin{array}{l}
x=a-U t-\frac{1}{k} e^{k(b-c)} \sin \left[k\left(a+c_{0} t\right)\right] \\
y=b-c+\frac{1}{k} e^{k(b-c)} \cos \left[k\left(a+c_{0} t\right)\right] \\
z=c+c \tan \alpha-\frac{\tan \alpha}{2 k} e^{2 k b_{0}}\left(1-e^{-2 k c(1+\cot \alpha)}\right)
\end{array}\right.
$$

where $k>0$ is a fixed wave number. $U$ is the uniform longshore current. The quantities $a, b, c$ do not express the initial coordinates of a particle, but are simply labeling variables serving to identify a particle. Our aim is to prove that the motion (3.1) is dynamically possible and that we can associate with it an expression for the hydrodynamical pressure $P$ such that the governing equations and boundary conditions are all satisfied. The resulting free surface of the water at $c=\left(b_{0}-b\right) \tan \alpha$ is the edge wave we are looking for. The map (3.1) is a diffeomorphism from the still water region $R$ to the water region bounded below by the rigid bed $z=0$ and above by the free water surface, which is parametrized by

$$
\left\{\begin{array}{l}
x=a-U t-\frac{1}{k} e^{k b(1+\tan \alpha)-k b_{0} \tan \alpha} \sin \left[k\left(a+c_{0} t\right)\right], \\
y=b(1+\tan \alpha)-k b_{0} \tan \alpha+\frac{1}{k} e^{k b(1+\tan \alpha)-k b_{0} \tan \alpha} \cos \left[k\left(a+c_{0} t\right)\right], \\
z=\left(b-b_{0}\right)(1+\tan \alpha) \tan \alpha-\frac{\tan \alpha}{2 k} e^{2 k b_{0}}\left(1-e^{2 k\left(b-b_{0}\right)(1+\tan \alpha)}\right),
\end{array}\right.
$$

with $a \in \mathbb{R}, b \leq b_{0}$ and $t \geq 0$. Indeed, observing that $(a, b, c) \mapsto(a, b-c, c)$ defines a diffeomorphism of $\mathbb{R}^{3}$, it suffices to show that the mapping

$$
\left(\begin{array}{l}
a \\
b^{\prime} \\
c
\end{array}\right) \mapsto\left\{\begin{array}{l}
a-U t-\frac{1}{k} e^{k b^{\prime}} \sin \left[k\left(a+c_{0} t\right)\right] \\
b^{\prime}+\frac{1}{k} e^{k b^{\prime}} \cos \left[k\left(a+c_{0} t\right)\right], \\
c+c \tan \alpha-\frac{\tan \alpha}{2 k} e^{2 k b_{0}}\left(1-e^{-2 k c(1+\cot \alpha)}\right),
\end{array}\right.
$$


with $a \in \mathbb{R}, b^{\prime} \leq b_{0}-c(1+\cot \alpha)$ and $t \geq 0$, is a diffeomorphism at every fixed value of $t$ (Constantin [12], Henry [19]). For notational convenience let us choose

$$
\xi=k(b-c), \theta=k\left(a-c_{0} t\right) .
$$

Then the Jacobian matrix of the transformation (3.1) is given by

$$
\left(\begin{array}{ccc}
\frac{\partial x}{\partial a} & \frac{\partial y}{\partial a} & \frac{\partial z}{\partial a} \\
\frac{\partial x}{\partial b} & \frac{\partial y}{\partial b} & \frac{\partial z}{\partial b} \\
\frac{\partial x}{\partial c} & \frac{\partial y}{\partial c} & \frac{\partial z}{\partial c}
\end{array}\right)=\left(\begin{array}{ccc}
1-e^{\xi} \cos \theta & -e^{\xi} \sin \theta & 0 \\
-e^{\xi} \sin \theta & 1+e^{\xi} \cos \theta & 0 \\
e^{\xi} \sin \theta & -1-e^{\xi} \cos \theta & z^{\prime}(c)
\end{array}\right) .
$$

The determinant of the matrix equals $\left(1-e^{2 \xi}\right) z^{\prime}(c)$. Due to the time-independence of the determinant, the fluid flow is volume-preserving, so that the Lagrangian form of the equation of continuity is fulfilled. From (3.1) we compute the velocity and acceleration of a particle as

$$
\left\{\begin{array} { l } 
{ u = \frac { D x } { D t } = - U - c _ { 0 } e ^ { \xi } \operatorname { c o s } \theta , } \\
{ v = \frac { D y } { D t } = - c _ { 0 } e ^ { \xi } \operatorname { s i n } \theta , } \\
{ w = \frac { D z } { D t } = 0 , }
\end{array} \quad \left\{\begin{array}{l}
\frac{D u}{D t}=k c_{0}^{2} e^{\xi} \sin \theta \\
\frac{D v}{D t}=-k c_{0}^{2} e^{\xi} \cos \theta \\
\frac{D w}{D t}=0
\end{array}\right.\right.
$$

Since

$$
\left(\begin{array}{c}
P_{a} \\
P_{b} \\
P_{c}
\end{array}\right)=\left(\begin{array}{ccc}
\frac{\partial x}{\partial a} & \frac{\partial y}{\partial a} & \frac{\partial z}{\partial a} \\
\frac{\partial x}{\partial b} & \frac{\partial y}{\partial b} & \frac{\partial z}{\partial b} \\
\frac{\partial x}{\partial c} & \frac{\partial y}{\partial c} & \frac{\partial z}{\partial c}
\end{array}\right)\left(\begin{array}{c}
P_{x} \\
P_{y} \\
P_{z}
\end{array}\right)
$$

and in view of (3.4), (2.1) can be written as

$$
\begin{gathered}
P_{x}=-\rho k c_{0}^{2} e^{\xi} \sin \theta, \\
P_{y}=\rho k c_{0}^{2} e^{\xi} \cos \theta-\rho g \sin \alpha, \\
P_{z}=-\rho g \cos \alpha,
\end{gathered}
$$

we have that (3.6) is the same as

$$
\begin{gathered}
P_{a}=-\rho\left(k c_{0}^{2}-g \sin \alpha\right) e^{\xi} \sin \theta, \\
P_{b}=\rho\left(k c_{0}^{2}-g \sin \alpha\right) e^{\xi} \cos \theta+\rho k c_{0}^{2} e^{2 \xi}-\rho g \sin \alpha, \\
P_{c}=-\rho\left(k c_{0}^{2}-g \sin \alpha\right) e^{\xi} \cos \theta-\rho k c_{0}^{2} e^{2 \xi}-\rho g \cos \alpha+\rho g(\cos \alpha+\sin \alpha) e^{2 k b_{0}} e^{-2 k c(1+\cot \alpha)} .
\end{gathered}
$$

Now, the gradient of the expression

$$
\begin{aligned}
& P=P_{0}+\rho \frac{\left(k c_{0}^{2}-g \sin \alpha\right)}{k} e^{\xi} \cos \theta+\frac{\rho g \sin \alpha}{2 k} e^{2 \xi}-\rho g\left(c \cos \alpha+\left(b-b_{0}\right) \sin \alpha\right) \\
& \quad-\frac{\rho g \sin \alpha}{2 k} e^{2 k b_{0}} e^{-2 k c(1+\cot \alpha)}
\end{aligned}
$$

with respect to the labeling variables is precisely the right-hand side of (3.10)-(3.12). Since the boundary condition (i) requires the pressure to be time independent on the surface, there can be no terms containing $\theta$ and the dispersion relation

$$
k c_{0}^{2}-g \sin \alpha=0
$$

must hold. Thus the propagation speed of the edge waves is $c_{0}=\sqrt{g \sin \alpha} / k$. This means that the uniform longshore current does not have an influence on the dispersion 
relation of the edge wave. Therefore the flow determined by (3.1) satisfies the governing equations (2.1). At the free surface $c=\left(b_{0}-b\right) \tan \alpha$, we have $P=P_{0}$ so that the dynamic boundary condition (i) is satisfied. The kinematic boundary condition (ii) at the free surface is also satisfied as at any instance the free surface (3.2) is the image of the still water surface $c=\left(b_{0}-b\right) \tan \alpha$ under (3.1) for all $t \geq 0$. Since the velocity of field is given by (3.4), the fluid velocity has no normal component to the beach when $z=0$, which proves (iii). The proof that (3.1) is an explicit solution to the governing equations for water waves with uniform longshore current on a sloping beach is completed.

\section{Discussion.}

4.1. Vorticity. In this section we will compute the vorticity of the flow (3.1). From (3.4) we get

$$
\left(\begin{array}{ccc}
\frac{\partial a}{\partial x} & \frac{\partial b}{\partial x} & \frac{\partial c}{\partial x} \\
\frac{\partial a}{\partial y} & \frac{\partial b}{\partial y} & \frac{\partial c}{\partial y} \\
\frac{\partial a}{\partial z} & \frac{\partial b}{\partial z} & \frac{\partial c}{\partial z}
\end{array}\right)=\left(\begin{array}{ccc}
\frac{1+e^{\xi} \cos \theta}{1-e^{2 \xi}} & \frac{e^{\xi} \sin \theta}{1-e^{2 \xi}} & 0 \\
\frac{e^{\xi} \sin \theta}{1-e^{2 \xi}} & \frac{1-e^{\xi} \cos \theta}{1-e^{2 \xi}} & 0 \\
0 & \frac{1}{z^{\prime}(c)} & \frac{1}{z^{\prime}(c)}
\end{array}\right)
$$

Thus the vorticity can be straightforwardly calculated as



We note that since the current is constant, it does not impact on the vorticity of the flow, and the prescription of the vorticity matches that of Constantin [12].

4.2. Run-up. The run-up is obtained by setting $z=0$ in (3.2) so that we have

$$
\left\{\begin{aligned}
x & =a-U t-\frac{1}{k} e^{k b_{0}} \sin \left[k\left(a+c_{0} t\right)\right] \\
y & =b_{0}+\frac{1}{k} e^{k b_{0}} \cos \left[k\left(a+c_{0} t\right)\right] \\
z & =0
\end{aligned}\right.
$$

The above formula shows that while the uniform longshore current influences the parametric representation of the run-up, it does not affect the amplitude of the edge wave.

\section{REFERENCES}

[1] P. H. Leblond and L. A. Mysak, Waves in the Ocean, Elsevier, 1978, 602 pp.

[2] G. G. Stokes, Report on recent researches in hydrodynamics, Rep. 16th Brit. Assoc. Adv. Sci. Southampton, Murray, London, 1846, 1-20.

[3] Horace Lamb, Hydrodynamics, reprint of the 1932 6th edition, with a foreword by R. A. Caflisch [Russel E. Caflisch]. Cambridge Mathematical Library, Cambridge University Press, Cambridge, 1993. MR 1317348 (96f:76001)

[4] F. Ursell, Edge waves on a sloping beach, Proc. Roy. Soc. London. Ser. A. 214 (1952), $79-97$. MR0050420(14,326f)

[5] Ulf Torsten Ehrenmark, Oblique wave incidence on a plane beach: the classical problem revisited, J. Fluid Mech. 368 (1998), 291-319, DOI 10.1017/S0022112098001888. MR1640061 (99j:76007)

[6] G. B. Whitham, Nonlinear effects in edge waves, J. Fluid Mech. 74 (1976), no. 2, 353-368. MR0421326(54 \#9331)

[7] H. Yeh, Nonlinear progressive edge waves: their instability and evolution, J. Fluid Mech. 152 (1985), 479-499.

[8] K. M. Mok and H. Yeh, On the mass transport of progressive edge waves, Physics of Fluids 11(10) (1999), 2906-2924.

[9] C. Yih, Note on edge waves in a stratified fluid, J. Fluid Mech. 24 (1966), 765-767. 
[10] Erik Mollo-Christensen, Allowable discontinuities in a Gerstner wave field, Phys. Fluids 25 (1982), no. 4, 586-587, DOI 10.1063/1.863802. MR658268 (84c:76074)

[11] F. Gerstner, Theorie der Wellen samt einer daraus abgeleiteten Theorie der Deichprofile (in German), Ann. Phys. 2 (1809), 412-445.

[12] Adrian Constantin, Edge waves along a sloping beach, J. Phys. A 34 (2001), no. 45, 9723-9731, DOI 10.1088/0305-4470/34/45/311. MR1876166 (2002j:76015)

[13] Mats Ehrnström, Joachim Escher, and Bogdan-Vasile Matioc, Two-dimensional steady edge waves. I. Periodic waves, Wave Motion 46 (2009), no. 6, 363-371, DOI 10.1016/j.wavemoti.2009.06.002. MR2598633 (2011i:76017)

[14] David Henry and Octavian G. Mustafa, Existence of solutions for a class of edge wave equations, Discrete Contin. Dyn. Syst. Ser. B 6 (2006), no. 5, 1113-1119 (electronic), DOI 10.3934/dcdsb.2006.6.1113. MR2224873 (2007a:76016)

[15] R. S. Johnson, Edge waves: theories past and present, Philos. Trans. R. Soc. Lond. Ser. A Math. Phys. Eng. Sci. 365 (2007), no. 1858, 2359-2376, DOI 10.1098/rsta.2007.2013. MR2329153 (2008f:76025)

[16] Raphael Stuhlmeier, On edge waves in stratified water along a sloping beach, J. Nonlinear Math. Phys. 18 (2011), no. 1, 127-137, DOI 10.1142/S1402925111001210. MR2786939(2012g:76033)

[17] Anca-Voichita Matioc, An exact solution for geophysical equatorial edge waves over a sloping beach, J. Phys. A 45 (2012), no. 36, 365501, 10, DOI 10.1088/1751-8113/45/36/365501. MR.2967917

[18] Adrian Constantin, Nonlinear water waves with applications to wave-current interactions and tsunamis, CBMS-NSF Regional Conference Series in Applied Mathematics, vol. 81, Society for Industrial and Applied Mathematics (SIAM), Philadelphia, PA, 2011. MR2867413

[19] David Henry, On Gerstner's water wave, J. Nonlinear Math. Phys. 15 (2008), suppl. 2, 87-95, DOI 10.2991/jnmp.2008.15.s2.7. MR2434727 (2009k:76020)

[20] A. Constantin, An exact solution for equatorially trapped waves, J. Geophys. Res. 117 (2012), C05029.

[21] A. Constantin and P. Germain, Instability of some equatorially trapped waves, J. Geophys. Res. 118 (2013), 1-9.

[22] Hung-Chu Hsu, An exact solution for equatorial waves, Monatsh. Math. 176 (2015), no. 1, 143-152, DOI 10.1007/s00605-014-0618-2. MR3296207

[23] A. Constantin, Some three-dimensional nonlinear equatorial flows, J. Phys. Oceanogr. 43 (2013), $165-175$.

[24] A. Constantin, Some nonlinear, equatorial trapped, non-hydrostatic, internal geophysical waves, J. Phys. Oceanogr. (2013), DOI 10.1175/JPO-D-13-0174.1.

[25] Hung-Chu Hsu, Some nonlinear internal equatorial flows, Nonlinear Anal. Real World Appl. 18 (2014), 69-74, DOI 10.1016/j.nonrwa.2013.12.011. MR.3176298

[26] Hung-Chu Hsu, An exact solution for nonlinear internal equatorial waves in the $f$-plane approximation, J. Math. Fluid Mech. 16 (2014), no. 3, 463-471, DOI 10.1007/s00021-014-0168-3. MR3247362

[27] Adrian Constantin, The trajectories of particles in Stokes waves, Invent. Math. 166 (2006), no. 3, 523-535, DOI 10.1007/s00222-006-0002-5. MR2257390 (2007j:35240)

[28] D. Henry, On the deep-water Stokes flow, Int. Math. Res. Not. 22 (2008), DOI 10.1093/imrn/rnn071.

[29] Adrian Constantin and Walter Strauss, Pressure beneath a Stokes wave, Comm. Pure Appl. Math. 63 (2010), no. 4, 533-557, DOI 10.1002/cpa.20299. MR.2604871 (2011b:76017)

[30] P. A. Howd, A. J. Bowen, and R. A. Holman, Edge waves in the presence of strong longshore currents, J. Geophys. Res. 97 (1992), 11357-11371.

[31] R. S. Johnson, A modern introduction to the mathematical theory of water waves, Cambridge Texts in Applied Mathematics, Cambridge University Press, Cambridge, 1997. MR.1629555 (99m:76017) 